20. MARTIN, S.A.; SEARS, D.L.; SEBO T.J. y cols.: "Smooth muscle neoplasms of the urinary bladder". Am. J. Surg. Pathol., 26: 292, 2002.

21. MARTINEZ, T.; VICENTE, R.; GUERRO, J.A.: "Leiomioma vesical: un nuevo caso a la casuística existente". Actas Urol. Esp., 19: 481, 1995.

22. LÓPEZ DE ALDA, A.; RODRÍGUEZ-MIÑÓN, J.L.; LITTON, M.: "Leiomioma vesical. Aportación de un nuevo caso". Arch. Esp. Urol., 44: 1145, 1991.

*23. GOMEZ, A.; SILMI, A.; FERNANDEZ, C. y cols.: "Leiomiomas de tracto urinario inferior". Arch. Esp. Urol., 44: 795, 1991.

*24. PÉREZ-CASTRO, E.; MARTÍNEZ-PIÑEIRO, J.A.; SILVA, J. y cols.: "Leiomyoma of the female urethra and bladder neck". Eur. Urol., 7: 46, 1981.

25. PÉREZ-CASTRO, E.: "Leiomiomas de tracto urinario inferior". Arch. Esp. Urol. (Comentario editorial), 44: 798, 1991.

Casos C línicos

Arch. Esp. Urol., 58, 9 (954-956), 2005

\section{LEIOMIOMA VESICAL EN PACIENTE VARÓN DE DIECISIETE AÑOS}

José Luis Moyano Calvo, María de los Ángeles Maqueda M arín, G uillermo Dávalos Casanova, Javier Sánchez de la Vega, Javier Giraldez Puig, Francisco Ignacio Huesa Ramírez, José Luis Maestro Durán, Antonio Ramírez Mendoza y Antonio Morales López.

Centro Urológico San Ignacio. Sevilla. España.

Resumen.- O BJETIVO: El leiomioma vesical es un tumor de presentación rara, cifrándose su frecuencia en la literatura en menos de un $1 \%$

Luis M oyano C alvo

C/ Eduardo Rivas, 3

41018 Sevilla. España.

e-mail: joseluismoyano@ya.com

Trabajo recibido: 14 de febrero 2005
MÉTODO: Presentamos el caso de un varón de 17 años, con dos pequeñas neoforma ciones vesicales descubiertas por hematuria y clínica irritativa miccional. RESULTADO S: Tras RTU de la lesión es diagnosticado de leiomioma vesical, sin recidiva en el periodo de seguimiento.

CON CLUSIO NES: El leiomioma vesical es un tumor benigno, por lo que hay que realizar cirugía 10 más conservadora posible. En la revisión realizada de las revistas urológicas españolas, es el caso más joven presentado hasta ahora.

Palabras clave: Leioma vesical. Tumor.

Summary- O BJEC TIVES: Bladder leiomyoma is a rare tumor, its frequency being estimated below $1 \%$.

METHODS: We report the case of a 17-year-old male patient presenting with hematuria and lower urinary tract irritative symptoms whose work up discovered two small bladder tumors.

RESULTS: After TUR of the lesions the diagnosis of bladder leiomyoma was established; no recurrences have appeared on follow-up.

CONCLUSIONS: Bladder leiomyoma is a benign tumor, therefore surgery should be the most conservative. Bibliographic review of the Spanish urologic journals show s that this is the youngest patient reported to date.

Keywords: Bladder leiomyoma. Tumor.

\section{INTRODUCCIÓN}

El leiomioma vesical, dada su escasa frecuencia, es habitualmente motivo de comunicación como nota clínica. En España se han comunicado al menos 32 leiomiomas vesicales, siendo la mayoría de ellos en mujeres (78\%) y pacientes adultos.

Presentamos el caso de un leiomioma vesical doble en varón de 17 años

\section{CASO CÚNICO}

Varón de diecisiete años sin antecedentes de interés que acude por polaquiuria, escozor miccional, tenesmo y hematuria autolimitada. 


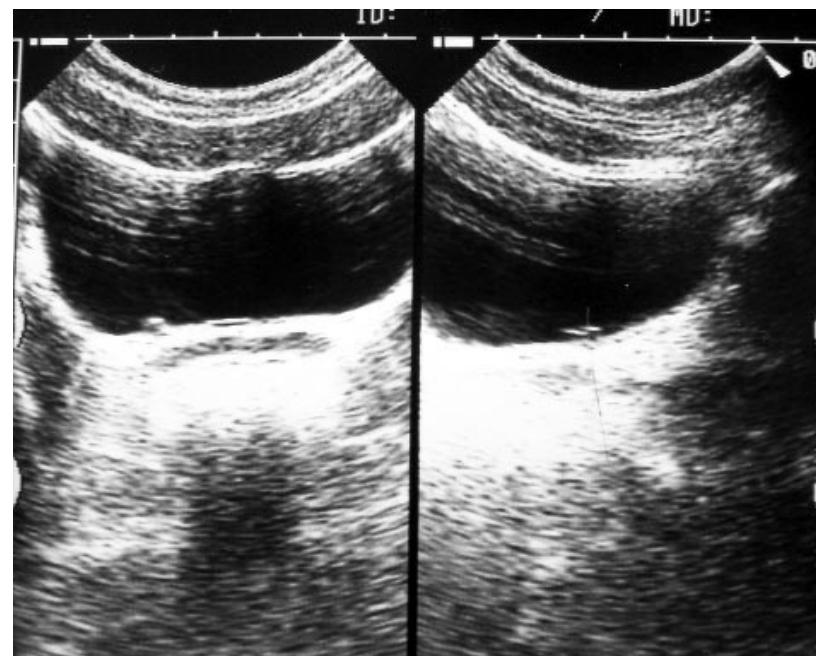

FIG URA 1. Lesión sólida con refuerzo sónico anterior en suelo vesical.

En la analítica de orina se aprecian 30-50 hematíes por campo. Urocultivo negativo.

Se practica ecografía abdominal observándose pequeña lesión de medio centímetro de diámetro, sólida con refuerzo hiperecogénico anterior (Figura 1).

Realizamos cistoscopia observándose en cara lateral derecha, por fuera de orificio ureteral dos pequeñas lesiones sobreelevadas, con mucosa aparentemente normal, similares a dos igloos.

Sospechándose lesión inflamatoria se prescribe tratamiento con A.I.N.E. durante diez días sin que desparezcan las lesiones, decidiéndose intervención quirúrgica.

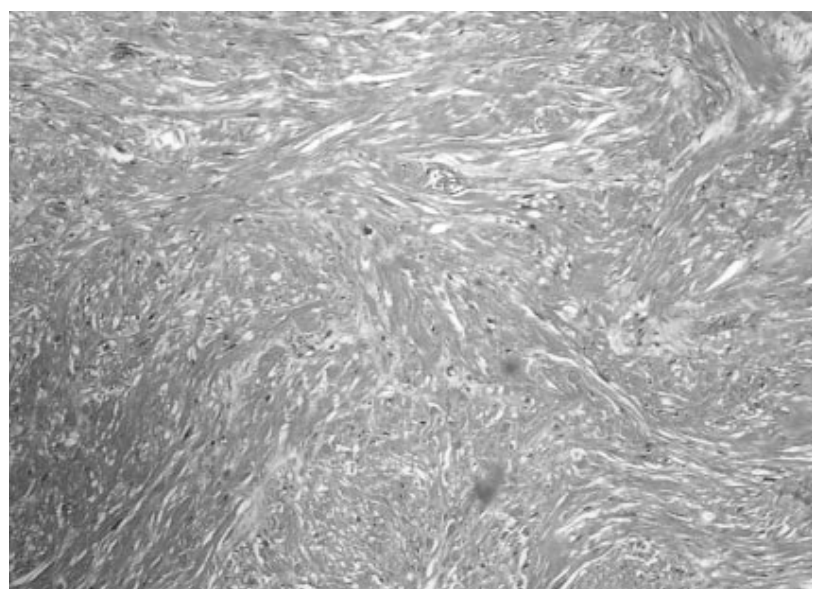

FIG URA 2. Haces entrecruzados de músculo liso sin alteraciones madurativas.
Se realiza RTU de ambas lesiones vesicales, siendo el informe anatomopatológico el de leiomioma vesical, describiendo la lesión como "proliferación de células fusiformes dispuestas en fascículos entrelazados con núcleos romos y citoplasma eosinófilo sin atipia, necrosis ni actividad mitótica significativa (Figura 2). Con el estudio inmunohistoquímico se demostró intensa positividad citoplasmática difusa para actina de músculo liso (Figura 3).

En el seguimiento efectuado al paciente no se ha apreciado recidiva de dichas lesiones.

\section{DISCUSIÓN}

El leiomioma vesical es un tumor de presentación rara, cifrándose su frecuencia en la literatura en menos de un $1 \%$, Es mucho más frecuente en la mujer y en al cuarta década (1-3).

Es el tumor mesotelial benigno más frecuente, aproximadamente un $47 \%$, seguido por el hemangioma. (4)

La localización más frecuente en el aparato urinario es en la cápsula renal, seguido de la vejiga. Dos terceras partes de los leiomiomas vesicales se localizan en el trígono

En vejiga se distinguen tres tipos según su localización $(4,5)$ :

- Extramurales

- Intramurales

- Submucosos, los más frecuentes, aproximadamente un $51-63 \%$

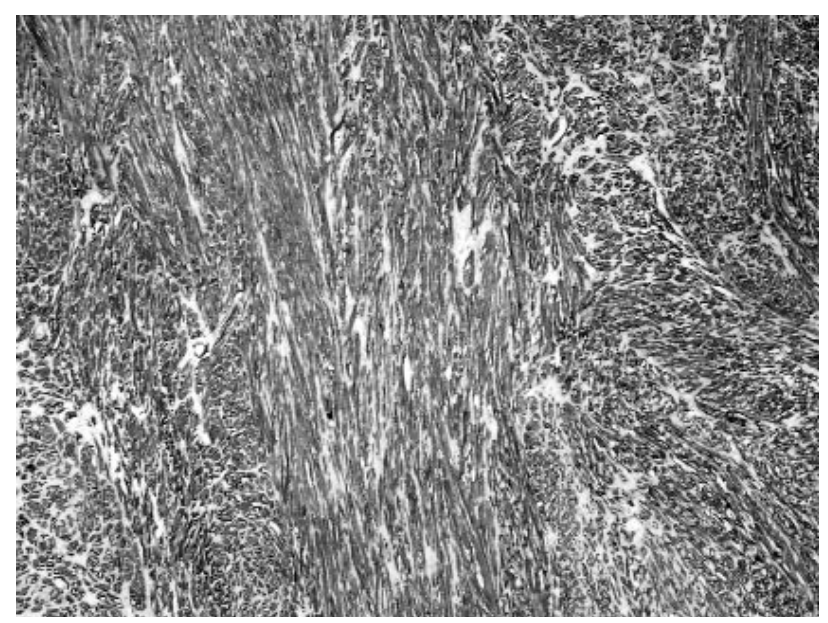

FIG URA 3. Estudio inmunohistoquímico. Positividad difusa para actina de músculo liso 
Existen varias teorías etiológicas (6):

- Inflamatoria

- Endocrina

- Disontogénica, considerándose restos del conducto de W olf o Müller

- Metaplasia perivascular

Clínicamente pueden cursar de manera asintomática, 0 bien con la aparición de síntomas urinarios irritativos (polaquiuria, urgencia, dolor suprapúbico) y/ u obstructivos (disuria, disminución calibre miccional). La hematuria se encuentra presente en el $20 \%$ de los casos (5)

La mejor técnica diagnóstica es la ecografía, que muestra habitualmente una lesión sólida, esférica, bien delimita$d a$, sin corrección parietal y con un refuerzo previo hiperecogénico que corresponde a la mucosa vesical intacta. (7)

En la cistoscopia son lesiones parietales sobreelevadas, lisas, bien delimitadas con mucosa de aspecto normal.

El tratamiento depende de su localización y tamaño. En los casos pequeños la Resección Transuretral (RTU) es suficiente, especialmente en los leiomiomas submucosos. En los leiomiomas mayores y en los leiomiomas extramurales se obtiene mejores resultados con cirugía abierta, bien enucleando el leiomioma cuando existe un buen plano de clivaje, bien realizando una cistectomía parcial. El abordaje puede realizarse transvaginal 0 transabdominal. $(5,8)$. En la literatura española consultada el 50\% de los leiomiomas se practico enucleación (tamaño medio de $4,8 \mathrm{~cm}$ ); el $27 \%$ se realizó cistectomía parcial (tamaño medio $5,5 \mathrm{~cm}$ ) y en el $23 \%$ se realizó RTU (tamaño medio $2,5 \mathrm{~cm}$ ).

No se ha demostrado su malignización y raramente recidivan como resultado de una exéresis incompleta.

En el estudio histopatológico la lesión esta constituida por una proliferación de células musculares lisas con núcleos pequeños, escasa mitosis y sin atipia.

En nuestro caso la lesión debutó con síndrome irritativo y hematuria, la presentación clínica más frecuente en el trabajo de Silva-Ramos (5), mostrando la imagen típica del leiomioma en la ecografía. Curiosamente tenia lesión doble, siendo más frecuente las lesiones únicas. Después de revisar las revistas urológicas españolas, no hemos encontrado publicado ningún caso en menores de 18 años, por lo que creemos que es el caso más joven comunicado hasta ahora en España.

\section{BIBUOGRAFÍA y LECTURAS RECOMEN DADAS (*lectura de interés y **lectura fundamental)}

1. TORRUBIA, F.J.; MONTANES, P.; SÁNCHEZ, M. y cols.: "Leiomioma vesical". Arch. Esp. Urol., 41: 17, 1988.

2. BLASCO, F.J.; SACTRISTAN, J. y cols.: "Características del leiomioma vesical en nuestro medio". Arch. Esp. Urol., 48: 987, 1995.

3. GOMEZ, A.; SILMI, A.; FERNANDEZ, C. y cols.: "Leiomiomas del tracto urinario inferior". Arch. Esp. Urol., 44: 795, 1991.

4. AlgABA, F.; MORENO, A.; TRIAS, I.: "Uropatología Tumoral". Pág.198. Pulso Ediciones SA. Barcelona, 1996.

*5. SILVA, M.; MASSÓ, P.; VERSOS, R. y cols.: "Leiomioma de vejiga. Análisis de agregación de 90 casos". Actas Urol. Esp., 27: 581, 2003.

6. RODRÍGUEZ, J.M.; TORROELLA, V.; RAMÍREZ, J. y cols: "Leiomioma vesical. Revisión de la literatura y presentación de un nuevo caso". Arch. Esp. Urol., 53: 167, 2000.

7. JIMENEZ, J.I.; LOZANO, F.; DE PABLO, A. y cols.: "Leiomioma de vejiga. A propósito de un caso". Actas Urol. Esp., 25: 223, 2001.

**8. TALLADA, M.; MARTINEZ, A.: "Leiomioma vesical”. En Patologías Excepcionales en Urología. Pág. 121-140. Luzan-5 Ediciones. Madrid 1998.

Casos C línicos

Arch. Esp. Urol., 58, 9 (956-959), 2005

\section{RABDOMIOSARCOMA DE CORDÓN ESPERMÁTICO EN EL ADULTO}

N uria Rodríguez García, Luis Lanes G onzález, Carlos Pascual M ateo y Antonio Berenguer Sánchez.

Servicio de Urología. Hospital Universitario de G etafe. Madrid España.

N uria Rodríguez García

Servicio de Urología

Hospital de G etafe

C tra. De Toledo. Km 12,500

$G$ etafe 28905 . M adrid. (España).

Trabajo recibido: 18 de febrero 2005 